\title{
Interaction energy calculation in Azaacenes type molecular crystals applied in organic electronics.
}

Sara S. Brito ${ }^{1}(\mathrm{PG})^{*}$, Luiz Antônio R. Junior ${ }^{1}(\mathrm{PQ})$, Pedro Henrique de Oliveira Neto $^{1}(\mathrm{PQ})$. sarahsantiagobrito@gmail.com

${ }^{1}$ Instituto de Física, Universidade de Brasília - 70.919-970, Brasília, Brasil.

Key words: Organic electronics, azaacenes, energy reorganization and interaction energy.

\section{Introduction}

The chemical structure of pentacene proposes wide applicability in organic electronic devices for molecules of the Azaacenes type. The manufacturing of electronic devices from the organic semiconductors is motivated by the increase of the capacity and performance of the systems, followed by the reduced cost of manufacture. In this work, we calculate the reorganization energy and the relative position of minimum energy in Tetraazapentacene crystals.

\section{Methodology}

The reorganization energy for electrons and holes was evaluated using the difference of energies between the molecules with its charged and neutral geometries of its charged and neutral species, following the well known four-point procedure. All calculations were performed using the Gaussian 09 program in density functional theory level with the B3LYP functional and 6-31G(d,p) base.

\section{Results and Discusion}

In figure 1 we have the tetraazapentacene molecule, the crystal constructed from the molecule is in figure 2 while in figure 3 we have the trimers extracted from the crystal.

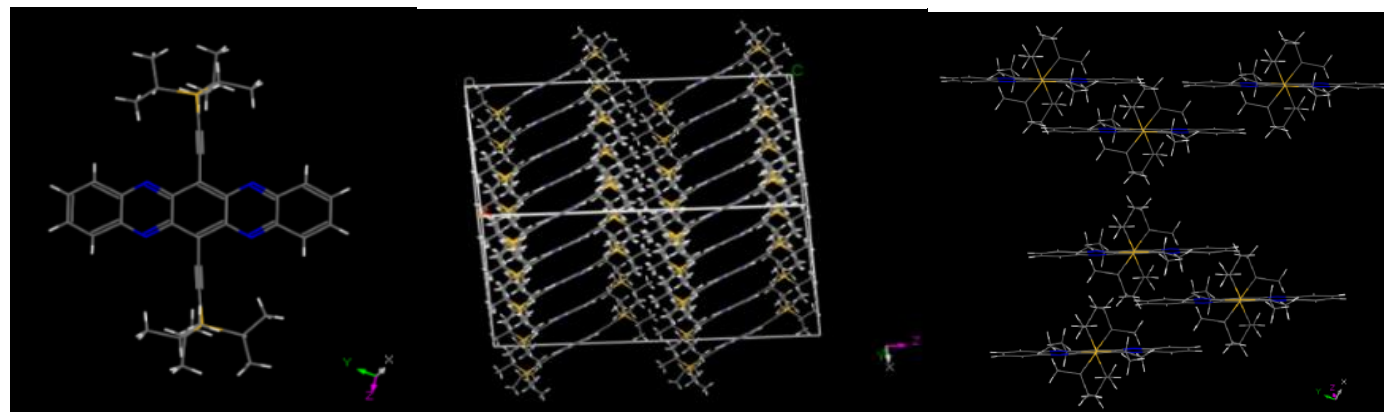

Figure 1

Figure 2

Figure 3

An important aspect of the crystal is the relative position of adjacent molecules with lowest energy configuration. In order to evaluate this geometry, we extracted trimers from the crystal and evaluated the energy of the system varying the position of the central molecule. Figure 4 shows the relative energy difference for each trimer also showing the position of minimum energy. 

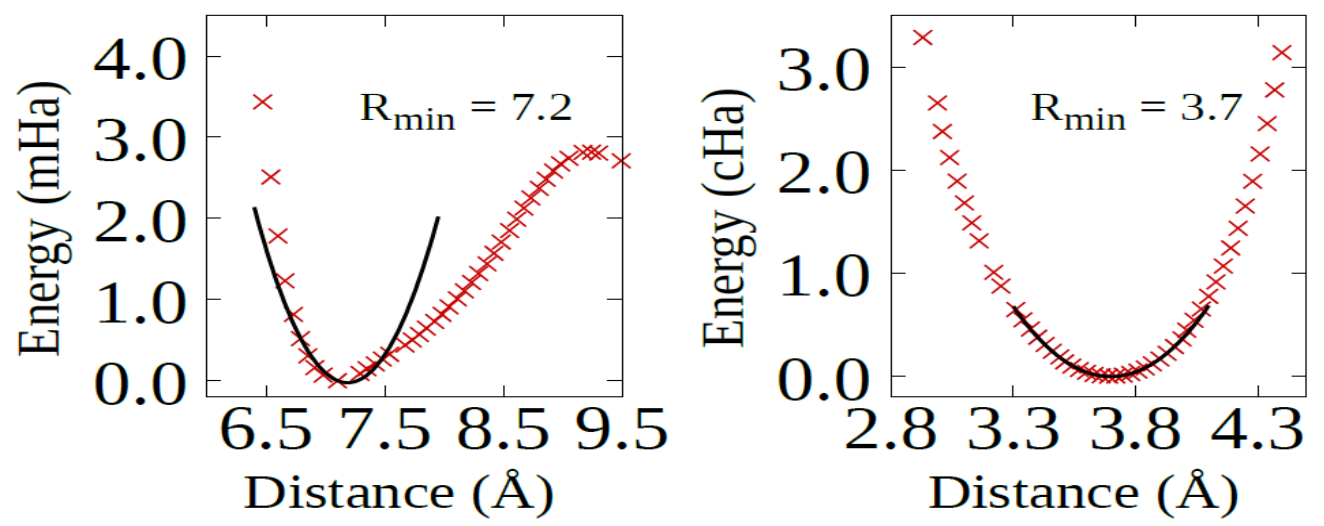

Figure 4

\section{Conclusions}

In this work, we study the transport properties of molecular crystals of pentacenes derivates known as Azaacenos. By studying the crystal morphology, we extracted the main trimes in which it was evaluated the energy for several relative configurations. By doing so, we estimate the position of minimum interaction for each configuration. Also, the reorganization energies were evaluated as $\lambda=204.43 \mathrm{meV}$ and $\lambda=211.05 \mathrm{meV}$ for electrons and holes respectively.

\section{Acknowledgments}

The authors gratefully acknowledge the financial support from CAPES, CNPq and FAP-DF.

\section{References}

1. F. S. Günther. S. Gemming. G. Seifert. Hopping Based Charge Transfer in Diketopyrrolopyrrole Based Donor-Acceptor Polymers: A Theoretical Study. J. Phys. Chem. C, Just Accepted Manuscript. JDOI: 10.1021/acs.jpcc.6b01310. Publication Date (Web): 19 Apr 2016. 